\title{
AOR
}

Selected Papers of \#AolR2020:

The 22nd Annual Conference of the

Association of Internet Researchers

Virtual Event / 13-16 Oct 2021

\section{THE SHAPE OF PLATFORM STUDIES: A MULTIDIMENSIONAL METHODOLOGICAL MODEL FOR A VICISSITUDINOUS CONCEPT}

Nathan Rambukkana

Wilfrid Laurier University

Gemma de Verteuil

Wilfrid Laurier University

\section{The Problematic}

The study of platforms is on the rise in communication studies, science and technology studies (STS), game studies, internet studies, and the study of human-machine communication (HMC). While originally platform studies emerged from hardware studies as an integrated attempt to study the hardware, software, code, marketing, and use of computational technologies-especially, early on, videogame consoles, but never limited to them-its use has been broadened to include the study of software platforms, such as social media sites, and their user affordances, algorithmic decision making, terms of service, background code environments, and embeddedness in neoliberal capitalism: selling user data, acting as advertising vehicles, etc. This holistic approach to studying technology encompasses everything from minute detail (such as how buttons are configured on a MINITEL console, or XBOX360 game controller; the way that privacy settings are displayed to Facebook users; the packaging of an Apple product), to the broad and situated socio-political context of corporations (such as the gender make up of Nintendo development teams, the political investments of the Twitter Board of Directors).

While a fruitful field with much work developed, there is a noticeable dearth of methodological theorising on the topic (Apperley and Parikka 2018), even as there are numerous theoretical explorations. How exactly does one do platform studies? After a deep dive into all the varietals of platform studies in the form of a graduate seminar, the authors concluded that the disparate approaches to studying platforms, while sometimes fought over as more-or-less appropriate or useful (e.g., Bogost and Montfort, 2009; Gillespie 2010), might be one of its core strengths. Which is to say that the vagueness of platform studies tracks the vagueness of platforms themselves (Gillespie 2010), and that this vicissitudinous nature might, in practice, be a feature not a bug.

Suggested Citation (APA): Rambukkana, N., de Verteuil, G. (2021, October). The Shape of Platform Studies: A Multidimensional Model for a Vicissitudinous Concept. Paper presented at AoIR 2021: The 22nd Annual Conference of the Association of Internet Researchers. Virtual Event: AolR. Retrieved from http://spir.aoir.org. 


\section{The Methodology: In Search of the Shape of Platform Studies}

We propose a multidimensional approach to platform studies, in which work may be located along at least three major axes: computational—sociotechnical, pragmaticcritical, and interpersonal-structural. These three dimensions of platform studies are combinable, provisional, and subject to extension. For example, platform studies work might be sociotechnical | critical | structural (for example van Dijck, Poell, and de Waal's work in The Platform Society (2018), or Srnicek's theorizing in Platform Capitalism (2017)). Work like this would foreground the societal and communicative implications of platforms with their computational aspects remaining relevant, but less of a focus. They would also centre critical aspects over pragmatic ones, a tone and line of attack that is often more structural than interpersonal.

Alternatively, it could be on the other end of this array-space and be computational | pragmatic | interpersonal such as the platform studies Montfort and Bogost build in Racing the Beam (2009), and some of their subsequent MIT Press Platform Studies series. The centre of attention in such studies is their computational affordances, which is to say their ability to be programed and/or play host to layers of further programmable platforms-like turtles, they may be platforms all the way down. The sociotechnical is still present but now rides shotgun and it is often (but not always, see for example, McKelvey 2011) the creative aspects of the sociotechnical that bear a secondary or joint focus. While critical aspects may still be present, the pragmatic focus of such studies can locate them closer to, for example, critical histories, policy, or regulatory workdepending on the genre of pragmatic focus. Similarly, while they may touch on structural issues, they more closely follow relationships and individuals: creative teams, innovators, insights, designs, competition, conflict, credit. With such studies, additional critical and structural aspects may be located by refocusing on the absences and gaps-Why are we only talking about the male or white creatives in a particular industry? Perhaps because the design teams were only white men, or perhaps because popular recounting attributes the labour of teams to particular individuals only: both worthy of critical reflection.

But work could also find itself at other locations within this triangulation, such as Danaher and McArthur's collection on Robot Sex (2018) which we argue is sociotechnical | critical | interpersonal, as it centres interpersonal ethics in its exploration of sex robotics as platform. While definitely critical, it breaks from more structurally focused work and speaks to relationships, individual needs, and desires, as well as society's perspectives on the same. You could also have work at different positions along a single axis, such as Gillespie's Custodians of the Internet (2018), that's focus on moderation practices could be described as sociotechnical | critical interpersonal-structural; which is to say somewhere between interpersonal and structural on the last coordinate, as it treats both the structural challenges of moderating social media at the scale of human society, and at the same time on moderation labour, its team dynamics, and interaction with publics very minutely.

Finally, we speak to how this model could be extensible and applied to other dimensions that could be identified or conceived of, e.g., technical-sociological, empirical - theoretical. We also speak to the model's limitations. Mathematical 
metaphors get strained a little here. A three-dimensional model has a certain intuitive recognition as a space, but when dimensions might be hot-swappable in ways that no longer track the "height | width | depth" paradigm, spatial metaphors could also fall apart. Similarly, the higher-mathematical forms present a challenge. If two dimensions form a matrix, and three an array or tensor, how do we talk about higher-dimensional methodological structures like $n$-dimensional arrays and tensors? Is tracking the mathematical form of something like a computational | critical | structural | theoretical exploration methodically fruitful, or belabouring? These potential limitations and lines of flight aside, we believe this model could be a path towards a more intentional, deliberate, and organized approach to platform studies. While the three dimensions offered up for discussion here cannot speak to the entirely of what platform studies is or could become, together and as a starting point these initial three define the shape of platform studies, track the work it has already done, and offer a solid framework and model for future investigations.

\section{References}

Apperley, Tom, and Jussi Parikka. 2018. "Platform Studies' Epistemic Threshold." Games and Culture 13, no. 4: 349-369.

Bogost, lan, and Nick Montfort. 2009. "Platform Studies: Frequently Questioned Answers." Digital Arts and Culture, December 12-15, 2009, Irvine, California, USA.

Danaher, John, and Neil McArthur, eds. 2017. Robot Sex: Social and Ethical Implications. Cambridge, MA: MIT Press.

Gillespie, Tarleton. 2010. "The Politics of Platforms." New Media \& Society 12, no. 3 : 347-364.

2018. Custodians of the Internet: Platforms, Content Moderation, and the Hidden Decisions that Shape Social Media. New Haven, CT: Yale University Press.

McKelvey, Fenwick. 2011. "FCJ-128 A Programmable Platform? Drupal, Modularity, and the Future of the Web" The Fibreculture Journal 18 (Trans). https://eighteen.fibreculturejournal.org/2011/10/09/fcj-128-programmableplatform-drupal-modularity-and-the-future-of-the-web/

Montfort, Nick, and lan Bogost. 2009. Racing the Beam: The Atari Video Computer System. Cambridge, MA: MIT Press.

Srnicek, Nick. 2017. Platform Capitalism. Cambridge, UK: Polity.

van Dijck, José, Thomas Poell, and Martijn de Waal. 2018. The Platform Society. Oxford, UK: Oxford University Press. 